\title{
AN ANALYSIS OF CASES OF DRUG-INDUCED LIVER INJURY REPORTED TO AN ADVERSE DRUG REACTION MONITORING CENTER
}

\author{
BALA SUBRAMANIAM, MEGHA SHAH*, CHETNA DESAI, JIGAR PANCHAL, SAMIDH SHAH
}

Department of Pharmacology, B.J. Medical College, Ahmedabad, Gujarat, India. Email: mkshah_80@yahoo.com

Received: 04 August 2020, Revised and Accepted: 09 September 2020

ABSTRACT

Objectives: Drug-induced liver injury (DILI) is a frequent cause of liver injury and acute liver failure .We aimed to analyze the cases of DILI reported over a period of 8 years to the adverse drug reaction (ADR) monitoring center (AMC) at our institution.

Methods: This observational retrospective study was conducted at the ADR monitoring center of a tertiary care hospital. Cases reported to the AMC, Pharmacovigilance Programme of India during the year 2011-2018 were analyzed as per the criteria used to analyze the ADRs.

Results: A total of 5448 ADRs were reported during the study period, of which 105 (2\%) were suspected to be DILI. The mean age of the patients with DILI was 39.26 years. Men (66.66\%) were more commonly affected than women (33.34\%). The most common drug groups causing DILI were antiretroviral (ART) (42.85\%) and antitubercular (ATT) (40\%). Most common single drug responsible for DILI was isoniazid (44.44\%) followed by atazanavir (28\%) and pyrazinamide (22.22\%). Increase in serum bilirubin was the most common DILI (64.75\%). About $79 \%$ of cases had a possible causality and $21 \%$ of cases had probable causal association with the suspected drugs. Majority of the ADRs (83\%) were not preventable and mild in severity (21\%). All ADR forms were complete in accordance with National Coordinating Center scale.

Conclusion: DILI is commonly observed in patients taking ART and ATT drugs for more than a month. Regular monitoring and assessment in these patients may help in preventing DILI and manage these ADRs.

Keywords: Drug-induced liver injury, Hepatotoxicity, Adverse drug reaction, AMC, Pharmacovigilance Programme of India.

(C) 2020 The Authors. Published by Innovare Academic Sciences Pvt Ltd. This is an open access article under the CC BY license (http://creativecommons. org/licenses/by/4. 0/) DOI: http://dx.doi.org/10.22159/ajpcr.2020.v13i11.39312

\section{INTRODUCTION}

Drug-induced liver injury (DILI) is a rare but potentially severe adverse drug reaction that should be considered in patients who develop laboratory criteria for liver injury secondary to the administration of a potentially hepatotoxic drug. Incidence of DILI in the west is reported to be $14 / 100,000$ patient-years [1,2]. Globally, the incidence is estimated to be between 10 and 15/10,000-100,000 persons exposed to prescription medications [2-4]. Accurate data of incidence of DILI in Indian population as such are not available. Although currently used liver parameters are sensitive in detecting DILI, they are neither specific nor able to predict the patient's subsequent clinical course [5]. Studies before the release of a drug are usually underpowered to detect rare side effects such as DILI and information on these is often obtained in the post-marketing phase. Drug-induced hepatotoxicity is the main reason for post-marketing regulatory decisions, including drug withdrawal [6]. Very few studies have been done in India for the evaluation of DILI. This study was aimed to determine the incidence and characteristics of DILI among the various adverse reactions reported to an AMC Pharmacovigilance Programme of India (PvPI) from a database over a duration of 8 years (2011-2018). The results would help guide the clinicians about the causal drugs, characteristics, and management of DILI in our health-care setup.

\section{METHODS}

This was an observational retrospective study, conducted at the Regional AMC PvPI, Department of Pharmacology, B.J. Medical College Ahmedabad, Gujarat, India. All the suspected adverse drug reactions (ADRs) were collected in Central Drugs Standard Control Organization approved spontaneous ADR reporting forms from treating consultants of various departments every month. The data from the database from the year 2011 to 2018 were evaluated and submitted through the VigiFlow to the Uppsala Monitoring Centre as a part of the routine functions of the AMC. All ADRs reported as DILI were identified from this database (January 2011-December 2018). The reference terms used for this purpose were raised liver enzymes, abnormal liver function test (LFT), hyperbilirubinemia, hepatotoxicity, icterus, and hepatitis.

\section{Inclusion criteria}

All suspected cases reported as DILI over the period of 8 years from 2011 to 2018 were evaluated for the study.

\section{Exclusion criteria}

The following criteria were excluded from the study:

- ADR reports that are incompletely filled.

- ADR reports not related to DILI.

\section{Informed consent}

No informed consent or ethical committee was obtained as the data were collected only from the computer database of the Pharmacology Department of B.J. Medical College.

Information regarding demographic details, alterations in LFT (serum glutamic oxaloacetic transaminase [SGOT], serum glutamic pyruvic transaminase [SGPT], and bilirubin levels), number of drugs prescribed, duration of therapy, drugs associated with DILI, dechallenge, and rechallenge was collected and entered into Microsoft Excel sheet version 2007.

Causality assessment was done using WHO-UMC scale and Naranjo's algorithm [7]. Severity assessment of reported DILI cases was carried out using Hartwig and Siegel scale. [8]. Preventability was assessed using the Shumock and Thornton criteria [9]. All the ADR forms were evaluated for completeness of ADR forms using National Coordinating Centre (NCC) Instrument [10]. 


\section{RESULTS}

Of the total 5448 ADRs, 105 cases of DILI were observed and analyzed. The DILI was most common in age group between 31 and 40 years. Mean age group of patients with DILI was 39.26 years. Male:female ratio was 2.5:1. SGOT, SGPT, and bilirubin levels were the investigations reported. The distribution of cases in each year from 2011 to 2018 is shown in Fig. 1.

The events reported as DILI were raised liver enzymes, abnormal LFT, hyperbilirubinemia, hepatotoxicity, icterus, and hepatitis (Table 1).

The latent period for DILI in most of the patients (48) was between 1 month and 1 year, between 1 week and 1 month in 15 patients, less than a week in 6 patients, and more than a year in 36 patients, as shown in Table 2.

Severity of the reported DILI cases was assessed by Hartwig and Siegel scale. About $79 \%$ of cases of DILI were mild in severity, no case of severe DILI was reported during the study period (Fig. 2).

Seriousness of reported DILI cases was evaluated and described in Table 3. No death was reported among patients with DILI during the study period. Majority (80\%) of DILI were non-serious while $18.05 \%$ required prolonged hospital stay.

Antiretroviral (ART) and antitubercular (ATT) drugs were common suspected drug groups implicated for DILI. Antibiotics, cyclooxygenase (COX)-2 inhibitors, steroids, and immunosuppressants were other groups causing DILI (Fig. 3). Analysis of individual drugs causing DILI are shown in Table 4

Common drug groups causing DILI were ATT and ART followed by antibiotics and COX-2 inhibitors. Among ART group, atazanavir $(45.45 \%)$ was the most common drug involved and among ATT group, isoniazid (57.1\%) was the most common drug involved in causing DILI. Most common single drug responsible for DILI was isoniazid (44.44\%) followed by pyrazinamide $(22.22 \%)$.

Among the DILI cases, 41 were caused by fixed-dose combinations (FDCs) and 64 cases were caused by a single drug. Atazanavir + ritonavir was the most common FDC causing DILI followed by zidovudine + nevirapine + lamivudine and tenofovir + lamivudine + efavirenz.

Among the reported DILI, $59 \%$ of cases were prescribed $<5$ drugs, whereas $46 \%$ of cases were prescribed more than 5 drugs. The causal

Table 1: Signs and symptoms of DILI recorded in ADR reporting forms $(n=105)$

\begin{tabular}{ll}
\hline Event & Number of ADRs (\%) \\
\hline Raised liver enzymes & $24(22.85)$ \\
Abnormal LFT & $4(3.80)$ \\
Hyperbilirubinemia & $37(35.23)$ \\
Hepatotoxicity & $8(7.6)$ \\
Icterus & $1(0.95)$ \\
Hepatitis & $28(26.67)$ \\
Total & 105 \\
\hline
\end{tabular}

DILI: Drug-induced liver injury, ADR: Adverse drug reaction, LFT: Liver function test

Table 2: Latent period for DILI in the study ( $n=105)$

\begin{tabular}{ll}
\hline Duration & Number of patients (\%) \\
\hline$<1$ week & $6(5.71)$ \\
1 week-1 month & $15(14.28)$ \\
1 month-1 year & $48(45.71)$ \\
1 year-6 years & $36(34.28)$ \\
Total & 105 \\
\hline
\end{tabular}

DILI: Drug-induced liver injury drugs were one in $36 \%$ of cases while it was more than one in $64 \%$ of DILI.

A total of $92 \%$ of cases of DILI were reported with oral medications and $6 \%$ of cases were reported with intravenous medication and $2 \%$ were reported with intramuscular medication.

Of the 105 cases of DILI, reported medications were prescribed for less than a week in $16 \%$, for 1 week to 1 month in $8 \%$, and for more than a month in $76 \%$ of cases.

Dechallenge of the suspected drug was done in 21 patients of DILI. No patients were rechallenged with the suspected drug. In 83 reported DILI, dose was not altered, drug was withdrawn in 21 patients and dose was reduced in 1 patient. About $56.19 \%$ of patients were taking

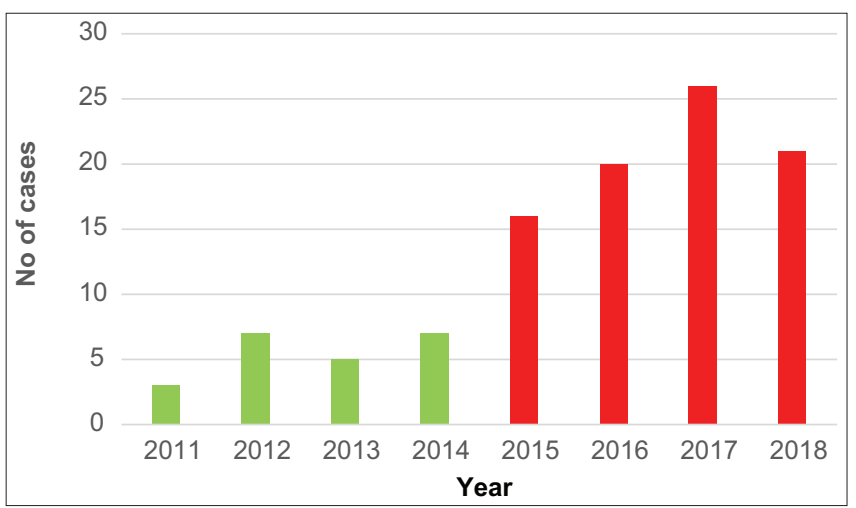

Fig. 1: Distribution of cases over the years $(n=105)$

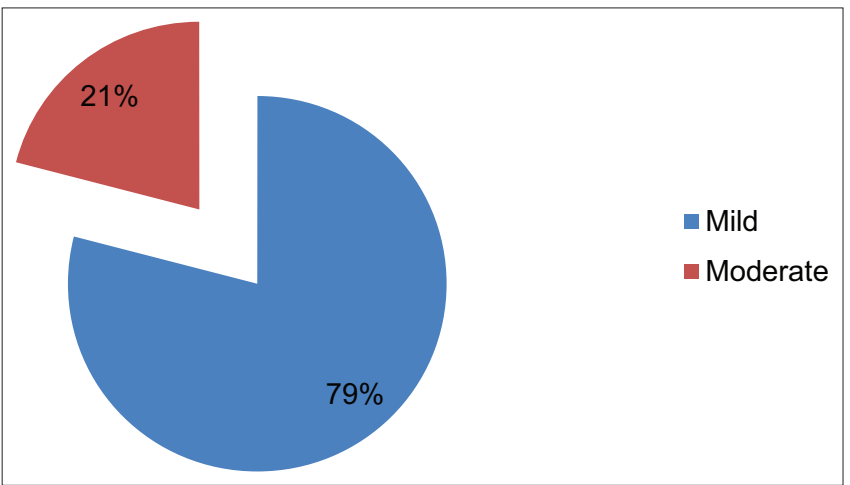

Fig. 2: Severity assessment of reported drug-induced liver injury at a tertiary care hospital $(n=105)$

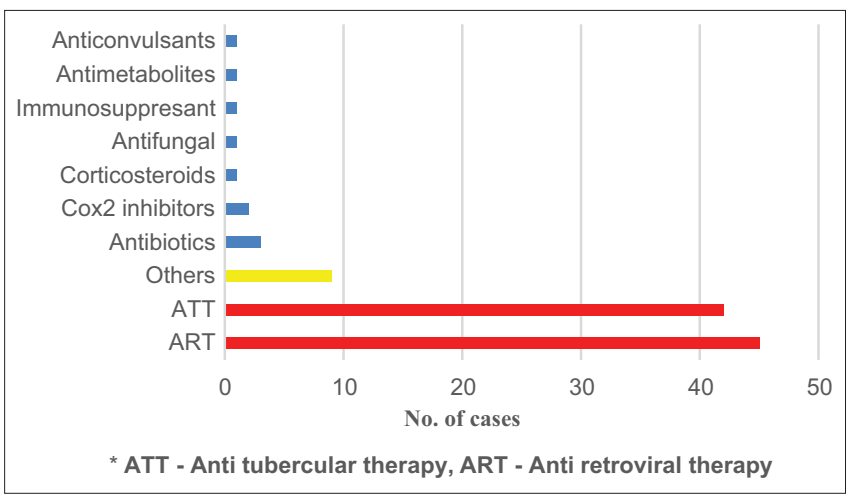

Fig. 3: Suspected drug groups causing drug-induced liver injury $(n=105)$ 
Table 3: Evaluation of seriousness of reported DILI cases

\begin{tabular}{llllllll}
\hline Not serious & Death & Life threatening & Prolonged hospitalization & Disability & Congenital anomaly & Intervention needed & Others \\
\hline $84(80 \%)$ & - & - & $19(18.05)$ & - & - & - & $2(1.09)$ \\
\hline DILI: Drug-induced liver injury & & & &
\end{tabular}

Table 4: Analysis of drugs reported to cause DILI at a tertiary care hospital

\begin{tabular}{|c|c|}
\hline Drugs & No. of cases (\%) \\
\hline Atazanavir & 6 \\
\hline Atazanavir + Ritonavir & 14 \\
\hline ZNL & 7 \\
\hline Tenofovir & 3 \\
\hline TL & 4 \\
\hline TLE & 7 \\
\hline TNL & 1 \\
\hline Abacavir & 1 \\
\hline Abacavir + Lamivudine & 1 \\
\hline Isoniazid & 20 \\
\hline Pyrazinamide & 10 \\
\hline Ethambutol & 1 \\
\hline HRE & 1 \\
\hline $\mathrm{HRZ}^{\#}$ & 2 \\
\hline $\mathrm{HRZ}^{\#} \mathrm{E}$ & 1 \\
\hline Rifampicin & 2 \\
\hline Ethionamide & 5 \\
\hline Clindamycin & $2(1.90)$ \\
\hline Ceftriaxone & $1(0.95)$ \\
\hline Vancomycin & $1(0.95)$ \\
\hline Methotrexate & $6(5.71)$ \\
\hline Disulfiram & $1(0.95)$ \\
\hline Etoricoxib & $1(0.95)$ \\
\hline Sulfasalazine & $1(0.95)$ \\
\hline Diclofenac sodium & $1(0.95)$ \\
\hline Infliximab & $1(0.95)$ \\
\hline Terbinafine & $1(0.95)$ \\
\hline Prednisolone & $1(0.95)$ \\
\hline Valproate & $1(0.95)$ \\
\hline Amiodarone & $1(0.95)$ \\
\hline Total & 105 \\
\hline
\end{tabular}

less than 5 drugs and $43.80 \%$ of patients were taking more than 5 drugs.

Causality analysis was done using WHO-UMC scale and Naranjo's algorithm and shown in Table 5.

Completeness of the ADR form was checked using NCC instrument and all the ADR forms were complete with a score of 14. The NCC score for completeness of ADR forms remained same throughout study period.

\section{DISCUSSION}

Drug-induced liver injury remains a challenge in modern hepatology. It is detected after multiple patients are exposed to the drugs. A wide variety of drugs was found to be associated with DILI in our study. A total of 29 different causal drugs for DILI were found

The present study analyses the DILI cases reported at a Regional AMC center as an adverse drug reaction over 8 years. The ADRs were reported from clinical departments of the Civil Hospital, Ahmedabad, a tertiary care hospital in Gujarat, India. The details of the ADRs were entered in the form structured by the Indian Pharmacopoeia Commission. A total of 105 cases of DILI were studied among the 5448 ADRs reported.

The investigations reported in the cases collected included serum bilirubin, SGOT, and SGPT. Patients having these enzyme levels raised
Table 5: Causality and preventability analysis of reported cases of DILI $(n=105)$

\begin{tabular}{lllll}
\hline Causality grade & Certain & Probable & Possible & Unlikely \\
\hline WHO-UMC scale & 0 & 22 & 83 & 0 \\
Naranjo's algorithm & 0 & 35 & 70 & NA \\
Preventability & Not preventable $-83 \%$ & Preventable - 17\% \\
\hline DILI: Drug-induced liver injury & &
\end{tabular}

more than 2 times of upper normal limit were considered to have druginduced liver injury.

Increase in serum bilirubin was the most common DILI followed by raised SGPT and SGOT. Among the different drug groups, antitubercular and anti-retroviral drugs were the most common drug groups involved accounting for total 87 cases out of the 105 cases of DILI. In the ART group, atazanavir was the most common drug involved, and in the ATT group, isoniazid was the most common drug involved.

Hepatic damage with ATT drugs in patients $<20$ years is rare but the incidence increases with age and ART drugs causing serious hepatic damage are rare but can be fatal [11].

Most of the drugs were prescribed for more than a month, implying a causal relationship between duration of therapy and DILI. Furthermore, many patients were taking more than 5 drugs which suggest that polypharmacy could be a factor in the etiology of DILI. The timeline from the year 2011 to 2018 showed similar trends in the drug groups causing DILI.

In a similar study done by Andrade et al. in Spain [12], in which 461 incidences of DILI submitted to the Spanish Registry were analyzed over a period of 10 years. The study stated that drug-induced hepatocellular jaundice has $11.7 \%$ chance of progressing to death or transplantation. In that study, antibiotics were the most common implicated drug group causing DILI followed by $\mathrm{H}_{2}$ antagonist and ATT drugs, whereas in our study, antitubercular drugs are the most common drugs causing DILI followed by ART drugs. The overall mean age group affected was 53 years in this study, whereas it was 39.26 years in our study.

Furthermore, a study done by Chalasani et al. in the United States of America [13], antibiotics were the most common drug group causing DILI. This study concluded that death due to DILI was significantly higher in patients with pre-existing liver disease or concomitant severe skin reactions compared to patients without. Moreover, in this study, the scores for likelihood of causal association with the suspected drug were definite in $22 \%$, highly likely in $43 \%$, probable in $18 \%$, possible in $13 \%$, and unlikely in $5 \%$, whereas in this study, the causal association with the suspected drug was possible in $79 \%$ and probable in $21 \%$ reports.

In another study done by De Valle et al. in Sweden [14], antibiotics were again the most common drug groups identified as causal agents causing DILI followed by NSAIDs. This study was conducted in an outpatient hepatology clinic. In this study, DILI cases constituted $6 \%$ of all outpatients and $3 \%$ of referrals and occurred more often in women. The mean age group of the affected patients in this study was 58 years. About $56 \%$ were female and $44 \%$ were male, whereas in our study, $71 \%$ were male and $29 \%$ were female. This difference may be due to higher number of male patients in our study. 
The reason for difference in drug groups involved in our study and other studies may be attributed to the difference in prevalence of disease patterns and the drug groups prescribed to treat them.

This study had its limitations. This was an observational retrospective study. Only the data that were available in the computer database were used. There are three basic types of liver injuries, hepatocellular presenting as acute hepatitis, cholestatic - presenting as cholestasis, and mixed [15]. However, the dominant liver injury type sometimes changes during illness [16]. However, we have not categorized these types in our study since relevant investigations were not available in all the patients at the time of analysis. Sample size was an added limitation of the study.

However, our study is one such of its kind as not much studies have been reported about DILI in India. Most of the DILI cases were not preventable $(83 \%)$, hence, proper counseling of the patients and caretakers regarding DILI before initiation of treatment would improve patient compliance.

\section{CONCLUSION}

This study offers a representative idea of the DILI cases in an Indian hospital. DILI is commonly seen in patients taking ATT and ART drugs. Hence, monitoring and vigilant assessment in these patients who require multidrug and long-term therapy may help improve patient management and prevent important ADRs. Moreover, creating awareness about the actual occurrence of DILI among prescribers, and counseling of patients and caretakers about triggering or aggravating factors for causation of DILI might help reduce their occurrence.

\section{ACKNOWLEDGMENT}

Authors would like to thank all the Residents of Pharmacology Department of B.J. Medical College and the staff of Civil Hospital, Ahmedabad.

\section{AUTHORS'CONTRIBUTIONS}

The study design and concept were done by Chetna Desai, Megha Shah, and Bala Subramaniam. I Bala Subramaniam wrote the paper, performed data collection, performed data analysis, drafted the manuscript, and contributed to the final manuscript. Megha Shah cowrote the paper, drafted the manuscript, and contributed to the final manuscript. Samidh Shah and Jigar Panchal designed the data analysis. Chetna Desai supervised the research and contributed to the final manuscript.

\section{CONFLICTS OF INTEREST}

Authors declare no conflicts of interest.

\section{AUTHORS' FUNDING}

The authors received no financial support for the research, authorship, and/or publication of this article.

\section{REFERENCES}

1. Sgro C, Clinard F, Ouazir K, Chanay H, Allard C, Guilleminet C, et al . Incidence of drug-induced hepatic injuries: A French population based study. Hepatology 2002;36:451-5.

2. Shapiro MA, Lewis JH. Causality assessment of drug induced hepatotoxicity: Promises and pitfalls. Clin Liver Dis 2007;11:477.

3. Holt M, Ju C. Drug induced liver injury. Handb Exp Pharmacol 2010;196:3-27.

4. Larrey D. Epidemiology and individual susceptibility to adverse drug reactions affecting the liver. Semin Liver Dis 2002;22:145.

5. Kullak-Ublick GA, Andrade RJ, Merz M, End P, Benesic A, Benesic A, et al. Drug-induced liver injury: Recent advances in diagnosis and risk assessment. Gut 2017;66:1154-64.

6. Bakke OM, Manocchia M, de Abajo F, Kaitin KI, Lasagna L. Drug safety discontinuations in the United States, and Spain from 1974-through 1993: A regulatory perspective. Clin Pharm Ther 1995;58:108-17.

7. Naranjo CA, Busto U, Sellars EM, Sandor P, Ruiz I, Roberts EA, et al. A method for estimating the probability of adverse drug reactions. Clin Pharmacol Ther 1981;30:239-45.

8. Hartwig SC, Siegel J, Schneider PJ. Preventability and severity assessment in reporting adverse drug reactions. Am J Hosp Pharm 1992:49:2229-32.

9. Schumock GT, Thornton JP. Focusing on the preventability of adverse drug reactions. Hosp Pharm 1992;27:538

10. Mahajan MM, Thatte UM, Gogtay NJ, Deshpande S. An analysis of completeness and quality of ADR reports at an adverse drug reaction monitoring centre in Western India. Perspect Clin Res 2018;9:123-6.

11. Alfred. Goodman and Gilman's the Pharmacological Basis of Therapeutics. $13^{\text {th }}$ ed. United States: McGraw-Hill Education; 2018. p. 1073-140.

12. Andrade RJ, Lucena MI, Fernandez MC, Pelaez G, Pachkoria K, García-Ruiz E, et al. Drug induced liver injury: An analysis of 461 incidences submitted to the Spanish registry over a 10 year period. Gastroenterology 2018; 17:795-804.

13. Chalasani N, Bonkovsky HL, Fontana R, Lee W, Stolz A, Talwalkar J, et al. Features and outcomes of 899 patients with drug induced liver injury: The DILIN prospective study. Gastroenterology 2015;148:1340-52.

14. De Valle MB, Klinteberg VA, Alem N, Olsson R, Bjornsson E. Druginduced liver injury in a Swedish university hospital out-patient hepatology clinic. Aliment Pharmacol Ther 2006;24:1187-95.

15. Hussaini SH, Farrington EA. Idiosyncratic drug induced liver injury: An overview. Expert Opin Drug Saf 2007;6:673-84.

16. Andrade RJ, Lucena MI, Kaplowitz N, Garcia-Munoz B, Borraz Y, Pachkoria K, et al. Outcome of acute idiosyncratic drug-induced liver injury: Long-term follow up in a hepatotoxicity registry. Hepatology $2006 ; 44: 1581-88$ 